\title{
Initial Condition Model from Imaginary Part of Action and the Information Loss Problem
}

\author{
H.B. Nielsen ${ }^{1 *}$ \\ 1 The Niels Bohr Institute, Copenhagen, Denmark
}

\begin{abstract}
We review slightly a work by Horowitz and Maldecena solving the information loss problem for black holes by having inside the blackhole - near to the singularity - a boundary condition, as e.g the no boundary proposal by Hartle and Hawking. Here we propose to make this boundary condition come out of our imaginary action model (together with Masao Ninomiya). This model naturally begins effectively to set up boundaries - whether it be in future or past! - especially strongly whenever we reach to high energy physics regimes, such as near the black hole singularity, or in Higgs producing machines as LHC or SSC. In such cases one can say our model predicts miracles. The point is that you may say that the information loss problem, unless you solve it in other ways, call for such a violation of time causality as in our imaginary action model!
\end{abstract}

*hbech@nbi.dk 


\section{Introduction}

The information loss problem [1] is essentially this:

1) From one point of view it seems that information falls into the black hole and

2) the information comming out with the Hawking radiation seems not so easy to get correlated with the infallen information.

But then it is not easy to get as t'Hooft [2] would like: the blackhole is just a resonance: you scatter some particles and some other particles come out connected by an ordinary S-matrix.

The point of the present talk is to look at the direction of solving this problem given by an article by Horowitz and Maldacena [3], in which they propose to use a fixing of the boundary conditions near or at the singularity inside the black hole. Let us immediately remark that such a direction of solving the problem is highly unconventional in the sense of having influence from the future, or should we say backward causation inside the black hole horizon.

\section{Review of Mathurs putting of the trouble}

We heard Samir Mathur[1] put the information loss problem roughly like this: In the usual Penrose diagram [5] for Black hole [4] the Horizon is a lightlike surface meaning in the Penrose diagram a lightlike directed line. We now imagine an extension of a curve of given external time t being the Schwarzhild coordinate but not in the perfect Schwarzhild solution extended but in one a little bit more realistic model including the very formation of the blackhole by some material falling in. Now we can find a surface, that is purely spacelike so that we could use it as description of a moment of time, a given special value of a time coordinate being in the far outside simply the time $t$ but which would at the end be seperated by the horizon.

The crux of the matter is that inside the horizon there are moment surfaces (space like surfaces, that could be taken as corresponding to a single value of a possible time coordinate) on which the information from the infallen material and the information correlated with the emitted Hawking radiation fall in widely different places. That is to say that the information about what falls in comes to one place at a certain "moment of time" while the Hawking radiation orginates at that moment of time from a far away region. Thus it seems against the principle of locallity to get the Hawking radiation correlated with the incomming material. But this correlation is what is needed if it should be so that the black hole were simply functioning as a normal resonance representing the scattering of the incomming material which then comes out again as Hawking radiation.

\section{Horowitz's and Maldacena's "solution"}

Since the problem of the information loss as here described is a matter of a problem with locality, one might think of having some form of violation of locality. Indeed Horowitz and Madacena [3] has a proposal for solving the information loss problem by such a violation. In fact the idea of Horowitz and Maldacena is that there could exist a boundary condition 
imposed as a law of nature at or close to the singularity inside the black hole. Such a boundary condition at a time later than the time for which it is relevant means a restriction of the future, an arrangement that the future shall be in a special or restricted state, and thus it a priori opens up the possibilty for backward causation, meaning that the future influences the past. Now it were in the Horowitz and Maldacena considerations concerning the black hole only in the inside the horizon space time region that the backward causation should take place. That should also be sufficient in order to solve the problem of the black hole information passing from the incomming matter to the outgoing Hawking radiation. In the outside the horizon region the reflection of the inside restriction at the singularity is that there comes a correlation between the infalling material and the outgoing Hawking radiation[6]. If we think of what happens on the above mentioned surface of events of a special moment of a certain time, we can see that the future restriction can impose a correlation between the far away regions with respectively the infalling stuff and the Hawking radiation related degrees of freedom. Such a restriction at the singularity could - Horowitz and Maldacena also allude to as the possibility - be due to the Hartle-Hawking no-boundary boundary condition[7]. To understand this idea of using a singularity based restriction "in the future" to provide the needed correlation it may be needed to have in mind that the Hawking radiation emmited related degrees of freedom fall into the black hole and finally end up in the singularity. Thus these with Hawking radiation related degrees of freedom get via the future restriction related to the infalling stuff degrees of freedom and so finally the Hawking radiation comming out ends up related to the degrees of freedom of the infalling stuff.

\section{Miracles are called for, unless only say string stars (fuzz balls)}

In the foregoing section we saw that Horowitz and Maldecena[3] could help on the information loss problem and thus make the black hole easier consistent with the developments as one usually expects it. I.e. we get as we expect the black hole to function as a normal resonance by introduction of restrictions at the singularity. But if we first let in the possibility of restrictions on the future as some law of nature then we have opened up for the possibility of getting miracles into the theory. In fact if we have restrictions on what the future shall be then we should expect to see that some features of the state of the universe should be predestined to some special fate. Such arrangements would seem like miraclulously special arrangements. The restrictions in the future would only come about typically by happenings which a priori would look so strange that we would consider them miracles. Thus the type of theory proposed is a theory with miracles. Now it were in the case of solving the black hole problem of information loss only inside the black hole inside the horizon - that were under the influence from future physics. Thus it were only inside the black hole that there were truly the need for the miracles, but if you allow them at all, it may at the end be difficult to keep them away from the outside region.

It should be mentioned that there may be other ways - although it looks difficult to solve the problem of information loss: In fact one can - and this is what stringtheory seems to deliver according to the talk of Samir Mathur[?] - imagine that a genuine black 
hole never truly forms, but that the collapse stops - if not before - in the last moment before a true black hole is formed. If truly a black hole were never formed, then of course the problems of information loss would not be relevant. In a way we can say that when as in Samir Mathurs picture the size of the string or string theory material forming the potential black hole remains of the size of the Schwarchild radius [4] even when the mass go so high that this Schwarchild radius has become very big, then it means that the black hole is not truly realized.

Such a keeping up the size of the stringtheory stuff to remain as big as the Schwarchild radius even when more and more stuff is being put on, can solve the information loss problem without need for any miracles.

\section{Ninomiya's and mine miracle model, imaginary part of action.}

The conclusion of the above discussion of the problem of black holes means that there is a call for a theory of the type with backward causation as suggested in the Horowitz and Maldacena article[3] reviewed above. This gives us the motivation and excuse for putting forward the model of Masao Ninomiya and myself [8]. I made an attempt to a popular presentation in a book of collections of talks on miracles [10] at AArhus University. Actually our ideas of influence from future are a bit related to old ideas of such influence mainly for the coupling constants [11] and for predestining humanity to make a new vacuum called the" vacuumbomb" [12].

This model [8][10] is characterized by having in it a prediction of initial conditions in principle. It is even so that this in principle predicted initial conditions are arranged so as to minimize a certain functional $S_{I}$ (history) depending on the history of the universe through all times from the beginning to the end, a functional being an integral over all space time, so that indeed it depends on both past and future. That is to say that the arrangement of the initial conditions to appear in our model depends also on the future and thus will appear as having prearrangement or backward causation in it. If for instance as we suggest it in our model that Higgs particles being produced will make $S_{I}$ (history) bigger than if they are not produced, then we should expect that there would be prearrangements occuring seemingly with the purpose of preventing the Higgs production. In fact one can approximately formulate the result of our predictions about the initial conditions by saying that they are adjusted so as to minimize the functional $S_{I}$ (history). That is to say the history of the universe will in our model be approximately selected among all the histories possible in accordance with the equations of motion as being that history which gives the smallest (i.e. most negative; it probably will be negative) $S_{I}($ history $)$-value.

This real quantity $S_{I}$ (history) which by being minimized determines the initial conditions is in our model actually the imaginary part of an -unusually - assumed complex action. That is to say our model consists actually in postulating that, contrary to what one usually takes it, the action for the development of the universe is fundamentally 
complex, i.e. of the form

$$
S(\text { history })=S_{R}(\text { history })+i S_{I}(\text { history }) .
$$

This is to be understood that the parameters in the action - such as coupling constants and mass squares (in the case we consider of a quantum field theory, the standard model say) - are taken to be complex, while the fields (or the dynamical variables) are taken as usual, i.e. real if they are real in the usual theory.

Since we now have an a bit unusual model with this complex action, meaning complex couplings and masses, we shall in principle make the model precise by setting up - or rather choose - that formalism in which we want simply to insert the complex action instead of the usual real action. It is honestly speaking a further assumtion in our model to choose just into which expression to insert the new complex action. We choose to do it in a formalism using the Feynman-Dirac-Wentzel path way integral but in slightly special way:

Usually one would use the Feynman-Wentzel-Dirac path way integration formalism [13][14] to calculate a development "matrix" giving the time development from one initial time $t_{i}$ to a final time $t_{f}$. Then the transition amplitude from one initial state $\mid i>$ to a final state $\mid f>$ is given as a functional integral

$$
<f\left|U\left(t_{i}, t_{f}\right)\right| i>=\int \exp \frac{i}{\hbar} S_{t_{i}-->t_{f}}(\text { path }) \mathcal{D} \text { path },
$$

where it is then understood that the action $S_{t_{i}->t_{f}}($ path $)$ is the integral of the Lagrangian - taken of course for the path being integrated over - over time from time $t_{i}$ to time $t_{f}$. Also it is understood that the field values of the path at the end points in time $t_{i}$ and $t_{f}$, let us call them $\phi\left(t_{i}\right)$ and $\phi\left(t_{f}\right)$ respectively, are to be integrated over with a weight given by the wavefuntion(al)s $\left\langle\phi\left(t_{i}\right) \mid i\right\rangle$ and $\left\langle\phi\left(t_{f}\right) \mid f\right\rangle$.

You would of course expect from the physical interpretation of the Feynaman path integral, that the weight of the contribution from the part of the integral where the fields at some time take the values in a certain interval should represent - in some way at least - the probability for the fields having taken their values in that interval. However, really the question as to what happens between the preparation of the state $\mid i>$ and the measurement of the final state $\mid f>$ cannot be answered because it would mean a new experiment to begin to measure on something in the intermediate time. It would be like in the Einstein Bohr discussion, if Einstein starts measuring through which of the slits in the double slit experiment the particle goes. Nevertheless Aharonov et al. [15] have discussed some weak measurements being performed in the intermediate time.

One could also for example make an expression for the average of an operator $O\left(t_{f}\right)$ at the time $t_{f}$ by means of the Feynman path way integral like this:

$$
\begin{array}{r}
<i\left|U\left(t_{i}->t_{f}\right)^{\dagger} O U\left(t_{i}->t_{f}\right)\right| i> \\
=\int \exp \frac{i}{\hbar} S_{t_{i-->t_{f}}}(\text { path }) O\left(\phi\left(t_{f}\right)\right) \mathcal{D} \text { path }\left(\int \exp \frac{i}{\hbar} S_{t_{i-->t_{f}}}\left(\text { path }^{\prime}\right) \mathcal{D}_{p a t h^{\prime}}\right)^{\dagger}
\end{array}
$$

where it is then to be explained that the boundaries for these two functional integrals at the end of time interval at $t_{i}$ should be integrated over and weighted with the wave 
function $<\phi\left(t_{i}\right) \mid i>$ and its complex conjugate $<i \mid \phi\left(t_{i}\right)>$. It is also understood that the path in the first factor and the path' in the second integral are to be identified at the time $t_{f}$,

$$
\phi\left(t_{f}\right)_{p a t h}=\phi\left(t_{f}\right)_{p a t h^{\prime}}
$$

Finally the operator $O\left(\phi\left(t_{f}\right)\right)$ should be understood as possibly depending even on the derivative of $\phi(t)$ derived w.r.t. to $t$ which is then identified with $t_{f}$.

Since in the usual case of the action being real the transition matrices as $U\left(t_{f}-->t_{3}\right)$ say is unitary we can even multiply in a product $U\left(t_{f}->t_{3}\right)^{\dagger} U\left(t_{f}->t_{3}\right)=1$, and thus we also write

$$
\begin{array}{r}
<i\left|U\left(t_{i}->t_{f}\right)^{\dagger} O U\left(t_{i}->t_{f}\right)\right| i> \\
=\int \exp \frac{i}{\hbar} S_{t_{i-->t_{3}}}(\text { path }) O\left(\phi\left(t_{f}\right)\right) \mathcal{D} p a t h\left(\int \exp \frac{i}{\hbar} S_{t_{i->t_{3}}}\left(\text { path }^{\prime}\right) \mathcal{D} p a t h^{\prime}\right)^{\dagger},
\end{array}
$$

where we now instead of at $t_{f}$ have the identification

$$
\phi\left(t_{3}\right)_{p a t h}=\phi\left(t_{3}\right)_{p a t h^{\prime}}
$$

That is to say that it does not matter for calculating the average of the operator $O\left(t_{f}\right)$ at $t_{f}$ whether we use the Feynman path integral with a time interval going up to one $t_{3}$ or another, so that we could if we would like take the choice of formulating it with taking $t_{3}$ to go say to infinity.

If we wanted we could even replace the initial time $t_{i}$ state $<i \mid$ by inserting a projection operator $|><i|$ and then putting also factor 1 from unitarity on the initial time side of the formula. In this way we could in the usual real action case obtain the expression for $<i\left|O\left(t_{f}\right)\right| i>$ of the form

$$
\begin{array}{r}
<i\left|U\left(t_{i}->t_{f}\right)^{\dagger} O U\left(t_{i}->t_{f}\right)\right| i> \\
\int \exp \left(\frac{i}{\hbar} S_{t_{0}->t_{3}}(\text { path })\right) “(|i><i|)\left(\phi\left(t_{i}\right)\right)^{\prime \prime} O\left(\phi\left(t_{f}\right)\right) \mathcal{D} \text { path } \\
\left(\int \exp \frac{i}{\hbar} S_{t_{0}->t_{3}}\left(\text { path }^{\prime}\right) \mathcal{D}_{\text {path }}\right)^{\dagger}
\end{array}
$$

where $t_{0}$ is a time that can be anything provided it is earlier than the time $t_{i}$ at which we have inserted the operator " $(|i><i|)\left(\phi\left(t_{f}\right)\right)^{\prime \prime}$ which is to replace the initial state $\mid i>$ used at first. Since now this expression does not in the real action case depend on the times $t_{0}$ and $t_{3}$ provided they are outside the time interval $\left[t_{i}, t_{f}\right]$, we could choose them to anything we would like as long as these times $t_{0}$ and $t_{3}$ are still outside. For instance we could take $t_{0}=-\infty$ and $t_{3}=+\infty$. We might even imagine as a slight generalization to insert several operators and think of replacing the special projection operator " $(|i><i|)\left(\phi\left(t_{i}\right)\right.$ " by any operator $O_{1}\left(\phi\left(t_{i}\right)\right)$ taken at the same time $t_{i}$ and thus write an expression like

$$
\int \exp \left(\frac{i}{\hbar} S_{t_{0}-->t_{3}}(\text { path })\right) O\left(\phi\left(t_{i}\right)\right) O\left(\phi\left(t_{f}\right)\right) \mathcal{D} \text { path }\left(\int \exp \left(\frac{i}{\hbar} S_{t_{0}->t_{3}}\left(p a t h^{\prime}\right)\right) \mathcal{D} p a t h^{\prime}\right)^{\dagger}
$$


as a suggestion for what we can use to extract information from a Feynaman path integral formulation. Note that this expression is quadratic in the Feynman path integral in the sence that it is a product of two Feynman path integrals, one with the dummy path being denoted path and one complex conjugated with the dummy path'.

Now the idea is in the case of a complex action $S=S_{R}+i S_{I}$ also to use this expression by postulating that this expression obtained by putting in combinations of operators into a Feynman path integral and then multiplying that by a complex conjugate path integral without the operator insertions to deliver expectation values for the to the operators associated quantities. That is to say we take it that our model is assumed to deliver the predictions obtained by being extracted from expressions of this type with $t_{0}$ and $t_{3}$ going to respectively minus and plus infinity.

While in the case of usually assumed real action model the extension with the time intervals outside the interval $\left[t_{i}, t_{f}\right]$ used is irrelevant, this is no longer true in the case of there being an imaginary part of the action $S_{I}$ (path). So in our model it becomes important that we decide to use the whole time axis from the beginning to the end of all times.

It is strictly speaking an assumption being added into our model that we postulate just this type of interpretation of our complex action model. We think, however, that just such an interpretation being based on using a Feynman-Wentzel-Dirac path integral extending a priori over all times from the beginning (big bang or whatever were the first moment, minus infinity likely) to the end of times is very reasonable. After all, if we should somehow think of the path integral as the fundamental theory beyond quantum mechanics then it would not be so nice to choose the time interval for evaluating the action to be put in the exponent of the path way integrand to depend on the choice of what we are to calculate, or even worse on some arbitrary choice. In the case of the real action when the arbitrary choice of the times $t_{0}$ and $t_{3}$ does not matter it would of course be o.k., but in the complex action where it would depend, the natural assumption will be to take the maximal time interval over which to integrate to be the one to use.

We think that it is also very natural to associate the expectation value of an operator $O$ at a time $t$ to be associated with the path at the time $\mathrm{t}$ and those components of the fields the development of which are described by the path and associated to the operator $O$. Thus we claim an interpretation of the form (12) to be quite reasonable.

\section{Significance of the imaginary part of the action}

Once we just have decided on an assumtion about the intepretation of our model using over all time Feynman path integrals - even without looking too much on the details alluded to in foregoing section of looking at expectaion values of operators and squaring the Feynman path integral - it should be rather unavoidable that only contributions to the Feynman path integral(s) from paths with the smallest (or rather most negative) imaginary action $S_{I}($ path) will have much significance. So it is almost obvious without much details that the history of the universe that will effectively be the one realized must be characterized by a very negative $S_{I}$ (history), the "smallest" $S_{I}$ (history). That it will be so follows from the simple fact that the Feynman path integral integrand has the 


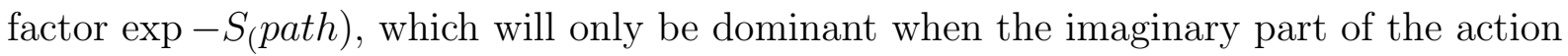
$S_{I}($ path $)$ is very negative.

Really we should have in mind that most likely the very long time intervals after $t_{f}$ "the future" and before $t_{i}$ "the past" will give big contributions to the imaginary part of the action, that will suppress most possible developments so much that essentially almost only one development, one history of the universe, comes to dominate. The best may actually be to think of performing a classical approximation. It is wellknown how in the Feynman path integral formulation one obtains the classical approximation as a saddle point approximation to the functional integral. Pedagogically - and to avoid a complicated discussion to extract at all a classical approximation, which is not so obvious at first - we may assume the imaginary part (at first) to be small. Then we would in first approximation be allowed to think upon the Feynman path integral as being in the classical approximation given by the saddle point contributions calculated at first as if we only had the real part $S_{R}($ path). This would mean quite usual classical solutions to the equations of motion would be all that would contributes in this approximation. But even a small imaginary part compared to the real part could give enormously big factors of the form $\exp -S_{I}$ (history) $/ \hbar$. We shall in fact not forget that in practice one expects $\hbar$ to be very small, so that for this reason already the exponent gets huge. When we think about that we have to do with integrals over time regions of the size of the whole lifetime of the universe, these imaginary action values for the whole life span of the unviverse will easily suppress almost all but one single classical solution. That is to say that even a in some sense small imaginary part would be far sufficient to drastically select almost only one sadle point contribution to survive being of significance.

We thus arrive at the first approximation description of the prediction of our model namely in a classical approximation:

First imagine calculate all the classical solutions using just the real part of the action. This delivers a set of all the classical solutions. Then calculate for each of these soltions (in practice of course we cannot do that, but think of it in principle), these possible histories history, the imaginary part of the action $S_{I}$ (history). Then our model predicts that just that history reahist which gets recognized as the realized one, the one that truly happens, is the one for which $S_{I}$ (reahist) is minimal.

This is what we could refer to as the formula "for the will of God" being

$$
S_{I}(\text { reahist }) \text { shall be minimal. }
$$

It should be had in mind that this type of determination of the initial state to be realized depending on an integral ${ }_{I}$ (history) which invloves all times, means that the way the universe developments has been started in a way depending on what could happen or not happen at much later times. But that then means that it would be like miracles, namely as if things have been prearranged in a statistically unexpected way, so as to just arrange that especially negative contributions to $S_{I}$ (hisory) get favoured by the selection of what really happens. I.e. negative imaginary part of the Lagrangian $L_{I}($ history $(t))$ (where history $(t)$ means the state and development derivatives in the history history at time equal to $t$ ) gets favoured to be big. One of our speculations to be discussed in section 7 is that production of Higgs particles should cause a relatively huge positive contribution to $L_{I}$ so that histories leading to Higgs production become disfavoured. 


\subsection{Really strong assumption, if we take action real}

First let us, however, now give an argument that, it would be very nice estetically to have the action not being restricted to be real, but rather to also have an imaginary part. Indeed we may simply think of the Feynmann-Wentzel path integral - even written only very abstractly without going in detail - written in the form

$$
\int \exp \left(\frac{i}{\hbar} S(\text { path })\right) \mathcal{D} \text { path }
$$

Now we come with the remark that it is completely obvious that the integrand $\exp \left(\frac{i}{\hbar} S(\right.$ path] $)$ is complex. There is namely even simply an $i$ present as an over all factor in the exponent. If we therefore take the point of view that the most fundamental and important quantity is the integrand rather than say the action itself, then we could say: if something should be assumed to be real rather than complex, then it should be this most fundamental quantity that should be assumed to be real.

To take the integrand to be real would of course be completely unacceptable, if one would have any connection to the usual theory. So the natural possibility is that there is no restricton to reality at all, so that both $S$ (path) and the integrand are allowed to be complex.

This argumentation may also be made more concrete by imagining that one would find some theory behind the Feyman Wentzel path way descripton of quantum mechanics, i.e. some model from which one derives quantum mechanics and arrive to a Feynman Wentzel path way formulation. Then if one would hope for the usual theory with the real action it would be a very delicate mechanism that would be needed to ever get the integrand become just a quantity of norm unity - as is what the real action means-. For example we attempted[?] such a derivation of quantum mechanics in the path way formulation and indeed did not at first find any reason why the integrand should be of norm unity.

\subsection{How to hide roughly the imaginary part of action}

At first it would look that our model with the complex action would lead to too many prearranged happenings to agree with what we observe; there would be too many "miracles" or "antimiracles" (repectively good or bad a priori unlikely events). Now, however, we have found some mechanism that might indeed help to reduce the predicted number of such at first unlikely arrangements in practice. Let us here in this discussion already accept the above mentioned classical approximation that we just have the effect of the imaginary part of the action, $S_{I}($ path $)$ in our model so that it just delivers the formula (13) to select the realized solution to the classical equations of motion.

The important point that brings down dramaticly the number of strange events, miracles or anti miracles, is that with the restriction from the equation of motions it is made troublesome to make too many miracles. If the initial state so to speak has to be adjusted to make certain special event at one moment of time then the degrees of freedom of this initial state are partly fixed by this arrangement and there is less freedom to adjust them to make - arrange for - more miracles. Thus it looks that the longer time the universe exists the more competition there will be about getting arrangements to each indivdual 
era of times. Only the "miracles" or "anti miracles" in the human history has a good chance to be spotted by poeple, and even then probably mainly the ones close to our own times, if we shall get aware of them. But since the universe has an age of the order of 13 milliard years already alone, the fact that there were $10^{8}$ human age periodes in even just the certainly existing time periode of for the universe, each arrangement would have to be shared by at least these $10^{8}$ periodes. Actually we believe from consideration of an action ansatz analogous the real part $S_{R}$ already known from phenomenology of the equations of motions, that it is likely that high energy scale physics contributes the most. When we think of the action as being written as a four dimensional space time integral $\int \mathcal{L} d^{4} x$ with the Lagrangian density having dimension mass to the fourth power, it should be obvious that in order to get a big contribution to the action from space time volume of a given size, we should involve physics with so high energies (per particles) involved as possible. Now the universe were of smaller size in the time shortly after big bang and the time scales of the eras were smaller so that this presumed higher contribution from the high energy scale being high is partly compensated for by smaller space time volume.

Nevertheless it is highly possible that a major contribution could have come to the imaginary part $S_{I}$ (history) from the era of inflation. One could even imagine that the as slow roll problem presented phenomenological call for a somewhat suspicially long time during which the inflaton field remained in a special region could be one of the "miracles" in our model. That should mean that because a special value for the inflaton field would give especially numerically high but negative imaginary part $L_{I}$ this value of the inflaton field would be (pre)arranged to be taken on over the biggest possible space time volume, the specially favoured value giving the very negative $L_{I}$. That might indeed favour what would look like a miraculously long stand in the inflation state.

If indeed some contribution from the time around big bang might dominate numerically

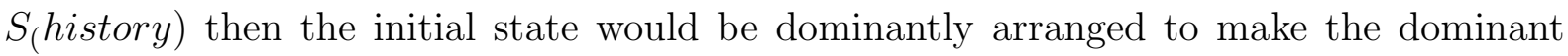
imaginary action contribution possibly most negative and then there would be less freedom of adjusting to make miracles at other times in the development of the universe. The point of course is that if first the initial state of the universe has been adjusted to give

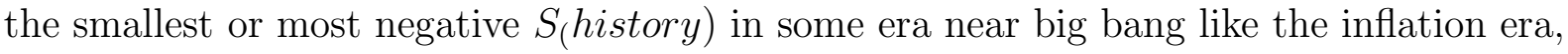
then there is less freedom to arrange miracles in later time. The equations of motion will namely determine what happens later once it has been determined with some "purpose" related to the inflation era, say.

So the hypotesis of a dominant era different from our own concerning the imaginary action $S_{I}$ (history) would help to reduce the number and degree of remarkableness of the miracles or anti miracles to be predicted to occur to day.

\section{$7 \quad$ Our prediction of the failure of LHC or other Higgs producing machines}

It is the suggested to be the major experimental test of our model of there being an imaginary part for the action, that production of many Higgs particles should be suppressed in the sense that machines destined to make big amounts of Higgs particles should have bad luck. We shall therefore now review this part of our model, i.e. the arguments for 
this bad luck comming out of our model for the big Higgs producing machines:

The most natural assumption about the order of magnitude of the imaginary part would be that we take the various coefficients in the expression for the Lagrangian density such as coupling constants and mass squares $\left(m_{h}^{2}\right.$ say) should have rather random phases of order unity. That would imply that we would expect the imaginary part $S_{I}$ and the real part $S_{R}$ of the action to be of similar order of magnitude for some random field development. For the special development, which the universe should perform in our model, and which is selected by minimizing the imaginary part it could be different. There is, however, one coefficient in the Standard Model Lagrangian density which requires a special consideration concerning the order of magnitude of real versus imaginary part, namely the mass square of the Higgs particle. The special point about this mass square of the Higgs particle is that it is a very wellknown mystery why the mass square of the Higgs particle defined in a renormalized way is so enormously small compared to the magnitude, which we would expect to have, namely the fundamental mass square scale of physics supposedly the Plack mass squared. This mystery we may call the "scale problem" - why so different scales ?! -. It would be even more a problem, if one would like to have a unified gauge theory like $S U(5)$ or $S O(10)$ or the like, since the unifying scales would also be far away from the Higgs mass scale - one would then even have the doublet triplet seperation problem-. It is really this scale problem that manifests itself by giving rise to the hierarchy problem: How to avoid that by each new perturbative correction to the Higgs VEV or Higgs renormalized mass quadratic divergences - supposedly cut off by some fundamental physics at the Plack scale - do not reshuffle the Higgs mass (square) by enourmous amounts recalling the scale problem mystery order by order again.

The part of the Higgs mass square coefficient for which we know the order of magnitude phenomenologically is the real part $\left.m_{H}^{2}\right|_{R}$ of this coefficient to the Higgs field squared $m_{H}^{2}$ in the Lagrangian density $\mathcal{L}=\ldots+m_{H}^{2} / 2 * \phi_{H}^{2}(x)+\ldots$, where we have split up the coefficient

$$
m_{H}^{2}=\left.m_{H}^{2}\right|_{R}+\left.i * m_{H}^{2}\right|_{I} .
$$

But now, if it is so mysterious, why the real part $\left.m_{H}^{2}\right|_{R}$ is so small compared to the Plack scale mass square (the square of the Planck mass), and we do not really understand yet the true mechanism for it being so small, then how can we know whether this "mysterious" mechanism works to also make the imaginary part of the Higgs mass square $\left.m_{H}^{2}\right|_{I}$ surprisingly small? Very likely it will actually not make also the imaginary part small, because what is truly what is small concerning the real part is not simply the bare real part, but rather the by several corrections modified - i.e. relative to that dressed or renormalized - real part. But renormalizing the imaginary part would likely be a quite different story so that a mysterious finetuning tuning the renormalized real part to be exceptionally small compared to the a priori expectation, the Planck mass square, would most likely not hit to make the imaginary part small. So we actually expect the imaginary part $\left.m_{H}^{2}\right|_{I}$ still to be of the order of the Planck mass square. But now from the point of view of the typical energy scales of say the LHC accelerator - a few $\mathrm{TeV}$ - the mysterious small Higgs mass and thus the real part $\left.m_{H}^{2}\right|_{R}$ is of a rather normal order of magnitude, while an imaginary part of Plack scale size would seem enourmously big! This then means that as soon as the imaginary part of the square of the Higgs mass comes in, it will completely dominate the present day contributions to the imaginary action. Now in the experiments 
we have so far studied, not even seeing the Higgs yet at all, the couplings and masses relevant have only been what came out of the dimensionless couplings/coefficients in the Standard model Lagrangian density and the Higgs vacuum expectation value. The latter is determined from the real part $\left.m_{H}^{2}\right|_{R}$ and thus the imaginary part would get so far no influence. There would as long as no Higgses are truly produced only be a constant vacuum contribution from the term $\left.m_{H}^{2}\right|_{I}|\phi(x)|^{2}$ to the imaginary Lagrangian density $\mathcal{L}(x)$.

Only when the Higgs field is modified relative to its uusual vacuum value VEV= $\left\langle\phi_{H}\right\rangle$ will the imaginary part come into play in a variable way. But that is typically the Higgs production and the existence of genuine Higgs particles flowing arround. We therefore expect that it is the flowing arround of produced Higgses, that will contribute the very likely very huge contribtuion to the $\mathcal{L}(x)$ and thus to $S_{I}$ (hitory]. Now presumably the appearance of geuine Higgs particles flowing arround is presumably a positive contribution to the imaginary action so that it would be disfavoured in the selection of the truly realized solution to the equations of motion to have Higgses arround; if it were namely instead very favoured we should already have Higgses all over.

Thus we now expect that it would make the imaginary part of the action $S_{I}$ (history) appreciably bigger (less negative) if in the history of the universe many Higgses come to exist, thus accelerators like SSC, the Tevatron, and the LHC producing Higgses should preferably for minimizing $S_{I}$ be avoided by not comming to work or quckly be stopped again once working. One should of course also then expect that cosmic rays should miraculously or somehow from the initial conditions of the universe have been arranged to produce as few Higgses as can easily be organized without spoiling too much the possibilities for the appropriate miracles in other eras so that their negative $S_{I^{-}}$-contributions are not too much reduced from what they can maximally be. But we humans have little understanding of how much cosmic rays there would have been under slightly different choices of the initial conditions, so we do not know if there should be a fine tuning of the initial conditions so as to make few or many cosmic radiation particles. Contrarily we have, however, good understandings of, that when one has built about the quarter of the tunnel of the planned SSC (= superconducting super collider)[?] in Texas, then there is apriori a very high expectation that there should soon be produced a lot of Higgs particles (if it exist at all of course as we assume here). Then it were really like an anti-miracle, when the Congress stopped the machine from being built and let the tunnel be only used for champignon growing or the like.

In our model we actually take this somewhat surprising fate of bad luck for the great SSC-project as an anti-miracle confirming our model. Also the accident of a bad connection stopping for soon a year the LHC just, when it were about to start functioning, we take as a symptom of our model! It should be stressed that our predictions about bad luck for Higgs producing accelerators were made after the bad fate of SSC, but before the accident at LHC that have delayed it by now soon a year! 


\section{Conclusion}

The main point of the presnt article were to call attention to that by the ideas of Horowitz and Maldacena [3] for solving the problem with black holes of correlating the infall information with the outgoing Hawking radiation a backward causation theory is called for. In competition with for instance the Hartle Hawking no-boundary postulate[7] replacing the singularity with a special condition - say no boundary - thereby imposing "final conditions" leading to backward causation we presnted "the imaginary part of action model" by Masa Ninomiya and myself[8]. The crux of this matter were, really as explained in the talk by Samir Mathur, the problem of getting the information from the infalling stuff into the black hole transfered to the outgoing Hawking radiation. This is a problem of causality - like the problem of tranfering information from one place to another place faster than with speed of light-. The problem would therefore possibly be avoided, if we have a theory with backward causation, so that future can influence past and therefore no causality principle can be truly valid. For phenomenological reasons it is of course needed that under "normal" conditions the amount of backward causation - or as we also refered to cases of backward causation, miracles or anti miracles - should be seldom. This is indeed the case both by thinking of Hartle Hawking no-boundary (mainly showing up in black holes, which are phenomenologically badly known) and in our "imaginary part of action model", in which it is though needed a somewhat speculative argumentation to argue that the cases of backward causation get so seldom as needed for agreement with dayly life experience. We think, however, that there is a good chanse that the restriction from the history of the universe having to obey the (classical) equations of motion (at least approximately) could impose so strong restrictions on the amount of backward causation or miracles or anti miracles that it would not disagree with present knowledge. In this way we want to claim that our model is viable so far.

\section{References}

[1] S. D. Mathur, "The information paradox: A pedagogical introduction," arXiv:0909.1038 [hep-th].

[2] Frontiers of Fundamental Physics Proceedings of the Sixth International Symposium Frontiers of Fundamental and Computational Physics, Udine, Italy, 26 September 2004 10.1007/1-4020-4339-2_4 B.G. SIDHARTH, F. HONSELL and A. DE ANGELIS

[3] Horowitz:2003he G. T. Horowitz and J. M. Maldacena, "The black hole final state," JHEP 0402 (2004) 008 [arXiv:hep-th/0310281].

[4] On the Means of Discovering the Distance, Magnitude, \&c. of the Fixed Stars, in Consequence of the D...; John Michell, Philosophical Transactions of the Royal Society of London, Vol. 74, (1784), pp. 35-57, Published by: The Royal Society

Schwarzschild, Karl (1916), "ber das Gravitationsfeld eines Massenpunktes nach der Einsteinschen Theorie", Sitzungsber. Preuss. Akad. D. Wiss.: 1892013196 and 
Schwarzschild, Karl (1916), "ber das Gravitationsfeld eines Kugel aus inkompressibler Flssigkeit nach der Einsteinschen Theorie", Sitzungsber. Preuss. Akad. D. Wiss.: 4242013434 . "Dark Stars (1783)". Thinkquest. http://library.thinkquest. org/25715/discovery/conceiving.htm\#darkstars. Retrieved 2008-05-28. Laplace; see Israel, Werner (1987), "Dark stars: the evolution of an idea", in Hawking, Stephen W. \& Israel, Werner, 300 Years of Gravitation, Cambridge University Press, Sec. 7.4

[5] \# d'Inverno, Ray (1992). Introducing Einstein's Relativity. Oxford: Oxford University Press. ISBN 0-19-859686-3. See Chapter 17 (and various succeeding sections) for a very readable introduction to the concept of conformal infinity plus examples. \# Frauendiener, Jrg. "Conformal Infinity". Living Reviews in Relativity. http://relativity.livingreviews.org/Articles/lrr-2004-1/index.html. Retrieved February 2, 2004. \# Carter, Brandon (1966). "Complete Analytic Extension of the Symmetry Axis of Kerr's Solution of Einstein's Equations". Phys. Rev. 141: 124220131247. doi:10.1103/PhysRev.141.1242. See also on-line version (requires a subscription to access) \# Hawking, Stephen; and Ellis, G. F. R. (1973). The Large Scale Structure of Space-Time. Cambridge: Cambridge University Press. ISBN 0-521-09906-4. See Chapter 5 for a very clear discussion of Penrose diagrams (the term used by Hawking \& Ellis) with many examples. \# Kaufmann, William J. III (1977). The Cosmic Frontiers of General Relativity. Little Brown \& Co. ISBN 0-316-48341-9. Really breaks down the transition from simple Minkowski diagrams, to Kruskal-Szekeres diagrams to Penrose diagrams, and goes into much detail the facts and fiction concerning wormholes. Plenty of easy to understand illustrations. A less involved, but still very informative book is his William J. Kaufmann (1979)). Black Holes and Warped Spacetime. W H Freeman \& Co (Sd). ISBN 0-7167-1153-2.

[6] Hawking, S. W. (1974). "Black hole explosions?". Nature 248 (5443): 30. doi:10.1038/248030a0. Hawking's first article on the topic Page, Don N. (1976). "Particle emission rates from a black hole: Massless particles from an uncharged, nonrotating hole". Physical Review D 13 (2): 198-206. doi:10.1103/PhysRevD.13.198. first detailed studies of the evaporation mechanism

[7] J. B. Hartle and S. W. Hawking, Phys. Rev. D 28 (1983) 2960. A. Vilenkin, arXiv:grqc/9812027.

[8] H. B. Nielsen and M. Ninomiya, "Nonexistence of irreversible processes in compact space-time," Int. J. Mod. Phys. A 22 (2008) 6227. H. B. Nielsen and M. Ninomiya, "Test of Influence from Future in Large Hadron Collider: A Proposal," arXiv:0802.2991 [physics.gen-ph]. H. B. Nielsen and M. Ninomiya, "Complex Action, Prearrangement for Future and Higgs Broadening," arXiv:0711.3080 [hep-ph].

H. B. Nielsen and M. Ninomiya, "Search for Future Influence from L.H.C," Int. J. Mod. Phys. A 23 (2008) 919 [arXiv:0707.1919 [hep-ph]].

H. B. Nielsen and M. Ninomiya, "Degenerate vacua from unification of second law of thermodynamics with arXiv:hep-th/0701018.

H. B. Nielsen and M. Ninomiya, "Future dependent initial conditions from imaginary part in lagrangian," arXiv:hep-ph/0612032. 
H. B. Nielsen and M. Ninomiya, "Trouble with irreversible processes in non-boundary postulate. and perfect match of equation of motions and number of fields," arXiv:hepth/0602186.

[9] H. B. Nielsen and M. Ninomiya, arXiv:hep-th/0601048.

H. B. Nielsen and M. Ninomiya, Int. J. Mod. Phys. A 21 (2006) 5151 [arXiv:hepth/0601021].

H. B. Nielsen and M. Ninomiya, "Unification of Cosmology and Second Law of Thermodynamics: Solving Cosmological Constant Problem, and Inflation," Prog. Theor. Phys. 116 (2007) 851 [arXiv:hep-th/0509205].

[10] Holger B. Nielsen, Model for Laws of Nature with Miracles, http://www.citebase.org/abstract?id=oai:arXiv.org:0811.0304, (2008)

[11] D. L. Bennett, C. D. Froggatt and H. B. Nielsen, "Nonlocality as an explanation for fine tuning in nature," (CITATION = C94-08-30);

D. L. Bennett, C. D. Froggatt and H. B. Nielsen, "Nonlocality as an explanation for fine tuning and field replication in nature," arXiv:hep-ph/9504294. (CITATION = HEP-PH/9504294;)

[12] D. L. Bennett, "Who is Afraid of the Past" ( A resume of discussions with H.B. Nielsen during the summer 1995 on Multiple Point Criticallity and the avoidance of Paradoxes in the Presence of Non-Locality in Physical Theories), talk given by D. L. Bennett at the meeting of the Cross-displiary Initiative at Niels Bohr Institute on September 8, 1995. QLRC-95-2. D. L. Bennett, "Multiple point criticality, nonlocality and fine tuning in fundamental physics: Predictions for gauge coupling constants gives alpha**(-1) $=136.8+-9$," arXiv:hep-ph/9607341. (CITATION = HEP-PH/9607341;) H. B. Nielsen and C. Froggatt, "Influence from the future," arXiv:hep-ph/9607375. (CITATION = HEP-PH/9607375;)

[13] Feynman and Hibbs,

Quantum Mechanics and Path Integrals (Hardcover) by Richard P. Feynman (Author), A. R. Hibbs (Author)

[14] Wentzel,

Physics Letters A Volume 324, Issues 2-3, 12 April 2004, Pages 132-138;

Salvatore Antoci and Dierck-E. Liebscher, Wentzel's Path Integrals,International Journal of Theoretical Physics Publisher Springer Netherlands ISSN 0020-7748 (Print) 1572-9575 (Online) Issue Volume 37, Number 1 / January, 1998 DOI 10.1023/A:1026628515300 Pages 531-535 Subject Collection Physics and Astronomy SpringerLink Date Wednesday, December 29, 2004;

International Journal of Theoretical Physics, Volume 37, Number 1, 1 January 1998 , pp. 531-535(5) 
[15] Y. Aharonov and L. Vaidman, Phys. Rev. A 41 (1990) 11. Y. Aharonov, T. Kaufherr and S. Nussinov, arXiv:0907.1666 [quant-ph].

Y. Aharonov, D. Z. Albert and C. K. Au, Phys. Rev. Lett. 47 (1981) 1765.

[16] D. Bennett, A. Kleppe and H. B. Nielsen, "Random dynamics in starting levels," http://www.slac.stanford.edu/spires/find/hep/www?irn=7560230SPIRES entry Prepared for 10th Workshop on What Comes Beyond the Standard Model, Bled, Slovenia, 17-27 Jul 2007 